\title{
Discourse Segmentation of Multi-Party Conversation
}

\author{
Michel Galley Kathleen McKeown \\ Columbia University \\ Computer Science Department \\ 1214 Amsterdam Avenue \\ New York, NY 10027, USA \\ \{galley, kathy\}ecs.columbia.edu
}

\author{
Eric Fosler-Lussier \\ Columbia University \\ Electrical Engineering Department \\ 500 West 120th Street \\ New York, NY 10027, USA \\ foslerdieee.org
}

\author{
Hongyan Jing \\ IBM T.J. Watson Research Center
}

Yorktown Heights, NY 10598, USA

hjing@us.ibm.com

\begin{abstract}
We present a domain-independent topic segmentation algorithm for multi-party speech. Our feature-based algorithm combines knowledge about content using a text-based algorithm as a feature and about form using linguistic and acoustic cues about topic shifts extracted from speech. This segmentation algorithm uses automatically induced decision rules to combine the different features. The embedded text-based algorithm builds on lexical cohesion and has performance comparable to state-of-the-art algorithms based on lexical information. A significant error reduction is obtained by combining the two knowledge sources.
\end{abstract}

\section{Introduction}

Topic segmentation aims to automatically divide text documents, audio recordings, or video segments, into topically related units. While extensive research has targeted the problem of topic segmentation of written texts and spoken monologues, few have studied the problem of segmenting conversations with many participants (e.g., meetings). In this paper, we present an algorithm for segmenting meeting transcripts. This study uses recorded meetings of typically six to eight participants, in which the informal style includes ungrammatical sentences and overlapping speakers. These meetings generally do not have pre-set agendas, and the topics discussed in the same meeting may or may not related.

The meeting segmenter comprises two components: one that capitalizes on word distribution to identify homogeneous units that are topically cohesive, and a second component that analyzes conversational features of meeting transcripts that are indicative of topic shifts, like silences, overlaps, and speaker changes. We show that integrating features from both components with a probabilistic classifier (induced with c4.5rules) is very effective in improving performance.

In Section 2, we review previous approaches to the segmentation problem applied to spoken and written documents. In Section 3, we describe the corpus of recorded meetings intended to be segmented, and the annotation of its discourse structure. In Section 4, we present our text-based segmentation component. This component mainly relies on lexical cohesion, particularly term repetition, to detect topic boundaries. We evaluated this segmentation against other lexical cohesion segmentation programs and show that the performance is state-of-theart. In the subsequent section, we describe conversational features, such as silences, speaker change, and other features like cue phrases. We present a machine learning approach for integrating these conversational features with the text-based segmentation module. Experimental results show a marked improvement in meeting segmentation with the incorporation of both sets of features. We close with discussions and conclusions.

\section{Related Work}

Existing approaches to textual segmentation can be broadly divided into two categories. On the one hand, many algorithms exploit the fact that topic segments tend to be lexically cohesive. Embodiments of this idea include semantic similarity (Morris and Hirst, 1991; Kozima, 1993), cosine similarity 
in word vector space (Hearst, 1994), inter-sentence similarity matrix (Reynar, 1994; Choi, 2000), entity repetition (Kan et al., 1998), word frequency models (Reynar, 1999), or adaptive language models (Beeferman et al., 1999). Other algorithms exploit a variety of linguistic features that may mark topic boundaries, such as referential noun phrases (Passonneau and Litman, 1997).

In work on segmentation of spoken documents, intonational, prosodic, and acoustic indicators are used to detect topic boundaries (Grosz and Hirschberg, 1992; Nakatani et al., 1995; Hirschberg and Nakatani, 1996; Passonneau and Litman, 1997; Hirschberg and Nakatani, 1998; Beeferman et al., 1999; Tür et al., 2001). Such indicators include long pauses, shifts in speaking rate, great range in F0 and intensity, and higher maximum accent peak. These approaches use different learning mechanisms to combine features, including decision trees (Grosz and Hirschberg, 1992; Passonneau and Litman, 1997; Tür et al., 2001) exponential models (Beeferman et al., 1999) or other probabilistic models (Hajime et al., 1998; Reynar, 1999).

\section{The ICSI Meeting Corpus}

We have evaluated our segmenter on the ICSI Meeting corpus (Janin et al., 2003). This corpus is one of a growing number of corpora with human-to-human multi-party conversations. In this corpus, recordings of meetings ranged primarily over three different recurring meeting types, all of which concerned speech or language research. ${ }^{1}$ The average duration is 60 minutes, with an average of 6.5 participants. They were transcribed, and each conversation turn was marked with the speaker, start time, end time, and word content.

From the corpus, we selected 25 meetings to be segmented, each by at least three subjects. We opted for a linear representation of discourse, since finer-grained discourse structures (e.g. (Grosz and Sidner, 1986)) are generally considered to be difficult to mark reliably. Subjects were asked to mark each speaker change (potential boundary) as either boundary or non-boundary. In the resulting annotation, the agreed segmentation based on majority

\footnotetext{
${ }^{1}$ While it would be desirable to have a broader variety of meetings, we hope that experiments on this corpus will still carry some generality.
}

opinion contained 7.5 segments per meeting on average, while the average number of potential boundaries is 770. We used Cochran's $Q$ (1950) to evaluate the agreement among annotators. Cochran's test evaluates the null hypothesis that the number of subjects assigning a boundary at any position is randomly distributed. The test shows that the interjudge reliability is significant to the 0.05 level for 19 of the meetings, which seems to indicate that segment identification is a feasible task. ${ }^{2}$

\section{Segmentation based on Lexical Cohesion}

Previous work on discourse segmentation of written texts indicates that lexical cohesion is a strong indicator of discourse structure. Lexical cohesion is a linguistic property that pertains to speech as well, and is a linguistic phenomenon that can also be exploited in our case: while our data does not have the same kind of syntactic and rhetorical structure as written text, we nonetheless expect that information from the written transcription alone should provide indications about topic boundaries. In this section, we describe our work on LCseg, a topic segmenter based on lexical cohesion that can handle both speech and text, but that is especially designed to generate the lexical cohesion feature used in the feature-based segmentation described in Section 5.

\subsection{Algorithm Description}

LCseg computes lexical chains, which are thought to mirror the discourse structure of the underlying text (Morris and Hirst, 1991). We ignore synonymy and other semantic relations, building a restricted model of lexical chains consisting of simple term repetitions, hypothesizing that major topic shifts are likely to occur where strong term repetitions start and end. While other relations between lexical items also work as cohesive factors (e.g. between a term and its super-ordinate), the work on linear topic segmentation reporting the most promising results account for term repetitions alone (Choi, 2000; Utiyama and Isahara, 2001).

The preprocessing steps of $L C s e g$ are common to many segmentation algorithms. The input document is first tokenized, non-content words are removed,

\footnotetext{
${ }^{2}$ Four other meetings failed short the significance test, while there was little agreement on the two last ones $(p>0.1)$.
} 
and remaining words are stemmed using an extension of Porter's stemming algorithm (Xu and Croft, 1998) that conflates stems using corpus statistics. Stemming will allow our algorithm to more accurately relate terms that are semantically close.

The core algorithm of LCseg has two main parts: a method to identify and weight strong term repetitions using lexical chains, and a method to hypothesize topic boundaries given the knowledge of multiple, simultaneous chains of term repetitions.

A term is any stemmed content word within the text. A lexical chain is constructed to consist of all repetitions ranging from the first to the last appearance of the term in the text. The chain is divided into subchains when there is a long hiatus of $h$ consecutive sentences with no occurrence of the term, where $h$ is determined experimentally. For each hiatus, a new division is made and thus, we avoid creating weakly linked chains.

For all chains that have been identified, we use a weighting scheme that we believe is appropriate to the task of inducing the topical or sub-topical structure of text. The weighting scheme depends on two factors:

Frequency: chains containing more repeated terms receive a higher score.

Compactness: shorter chains receive a higher weight than longer ones. If two chains of different lengths contain the same number of terms, we assign a higher score to the shortest one. Our assumption is that the shorter one, being more compact, seems to be a better indicator of lexical cohesion. ${ }^{3}$

We apply a variant of a metric commonly used in information retrieval, TF.IDF (Salton and Buckley, 1988), to score term repetitions. If $R_{1} \ldots R_{n}$ is the set of all term repetitions collected in the text, $t_{1} \ldots t_{n}$ the corresponding terms, $L_{1} \ldots L_{n}$ their respective lengths, ${ }^{4}$ and $L$ the length of the text, the adapted metric is expressed as follows, combining frequency $\left(\right.$ freq $\left.\left(t_{i}\right)\right)$ of a term $t_{i}$ and the compactness of its underlying chain:

$$
\operatorname{score}\left(R_{i}\right)=\operatorname{freq}\left(t_{i}\right) \cdot \log \left(\frac{L}{L_{i}}\right)
$$

\footnotetext{
${ }^{3}$ The latter parameter might seem controversial at first, and one might assume that longer chains should receive a higher score. However we point out that in a linear model of discourse, chains that almost span the entire text are barely indicative of any structure (assuming boundaries are only hypothesized where chains start and end).

${ }^{4}$ All lengths are expressed in number of sentences.
}

In the second part of the algorithm, we combine information from all term repetitions to compute a lexical cohesion score at each sentence break (or, in the case of spoken conversations, speaker turn break). This step of our algorithm is very similar in spirit to TextTiling (Hearst, 1994). The idea is to work with two adjacent analysis windows, each of fixed size $k$. For each sentence break, we determine a lexical cohesion function by computing the cosine similarity at the transition between the two windows. Instead of using word counts to compute similarity, we analyze lexical chains that overlap with the two windows. The similarity between windows ( $A$ and $B$ ) is computed with: ${ }^{5}$

$$
\operatorname{cosine}(A, B)=\frac{\sum_{i} w_{i, A} \cdot w_{i, B}}{\sqrt{\sum_{i} w_{i, A}^{2} \sum_{i} w_{i, B}^{2}}}
$$

where

$w_{i, \Gamma}=\left\{\begin{array}{cc}\operatorname{score}\left(R_{i}\right) & \text { if } R_{i} \text { overlaps } \Gamma \in\{A, B\} \\ 0 & \text { otherwise }\end{array}\right.$

The similarity computed at each sentence break produces a plot that shows how lexical cohesion changes over time; an example is shown in Figure 1. The lexical cohesion function is then smoothed using a moving average filter, and minima become potential segment boundaries. Then, in a manner quite similar to (Hearst, 1994), the algorithm determines for every local minimum $m_{i}$ how sharp of a change there is in the lexical cohesion function. The algorithm looks on each side of $m_{i}$ for maxima of cohesion, and once it eventually finds one on each side ( $l$ and $r$ ), it computes the hypothesized segmentation probability:

$$
p\left(m_{i}\right)=\frac{1}{2}[\operatorname{LCF}(l)+\operatorname{LCF}(r)-2 \cdot \operatorname{LCF}(m)]
$$

where $\operatorname{LCF}(x)$ is the value of the lexical cohesion function at $x$.

This score is supposed to capture the sharpness of the change in lexical cohesion, and give probabilities close to 1 for breaks like sentence 179 in Figure 1.

Finally, the algorithm selects the hypothesized boundaries with the highest computed probabilities. If the number of reference boundaries is unknown, the algorithm has to make a guess. It computes the

\footnotetext{
${ }^{5}$ Normalizing anything in these windows has little effect, since the cosine similarity is scale invariant, that is $\operatorname{cosine}\left(\alpha x_{a}, x_{b}\right)=\operatorname{cosine}\left(x_{a}, x_{b}\right)$ for $\alpha>0$.
} 


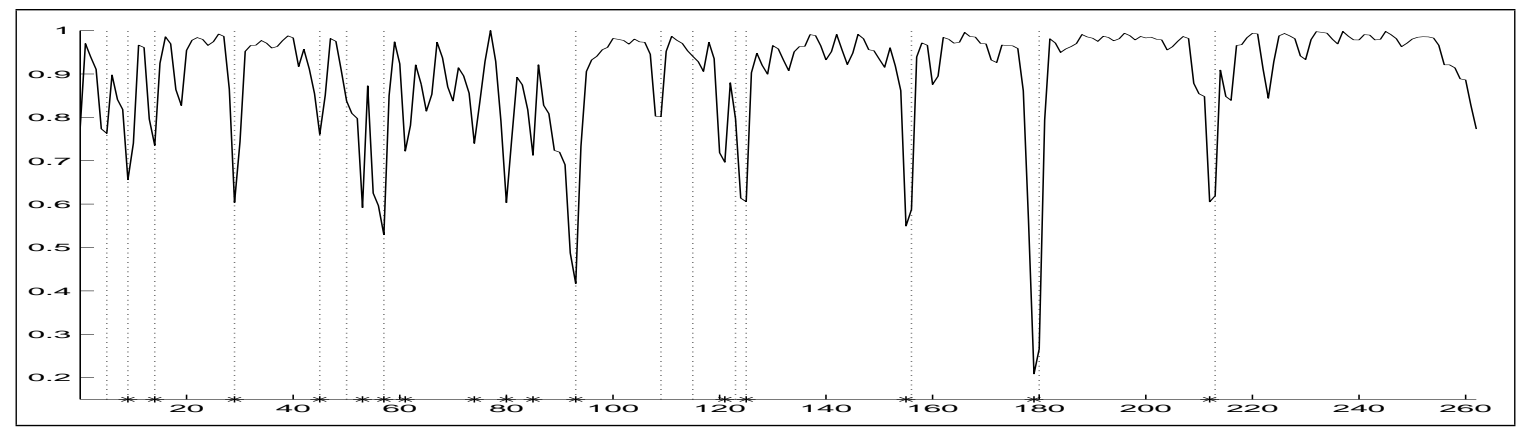

Figure 1: Application of the LCseg algorithm on the concatenation of 16 WSJ stories. Numbers on the $\mathrm{x}$-axis represent sentence indices, and $\mathrm{y}$-axis represents the lexical cohesion function. The representative example presented here is segmented by $L C s e g$ with an error of $P_{k}=15.79$, while the average performance of the algorithm is $P_{k}=15.31$ on the WSJ test corpus (unknown number of segments).

mean and the variance of the hypothesized probabilities of all potential boundaries (local minima). As we can see in Figure 1, there are many local minima that do not correspond to actual boundaries. Thus, we ignore all potential boundaries with a probability lower than $p_{\text {limit }}$. For the remaining points, we compute the threshold using the average $(\mu)$ and standard deviation $(\sigma)$ of the $p\left(m_{i}\right)$ values, and each potential boundary $m_{i}$ above the threshold $\mu-\alpha \cdot \sigma$ is hypothesized as a real boundary.

\subsection{Evaluation}

We evaluate $L C s e g$ against two state-of-the-art segmentation algorithms based on lexical cohesion (Choi, 2000; Utiyama and Isahara, 2001). We use the error metric $P_{k}$ proposed by Beeferman et al. (1999) to evaluate segmentation accuracy. It computes the probability that sentences $k$ units (e.g. sentences) apart are incorrectly determined as being either in different segments or in the same one. Since it has been argued in (Pevzner and Hearst, 2002) that $P_{k}$ has some weaknesses, we also include results according to the WindowDiff (WD) metric (which is described in the same work).

A test corpus of concatenated ${ }^{6}$ texts extracted from the Brown corpus was built by Choi (2000) to evaluate several domain-independent segmentation algorithms. We reuse the same test corpus for our evaluation, in addition to two other test corpora we constructed to test how segmenters scale across genres and how they perform with texts with various

\footnotetext{
${ }^{6}$ Concatenated documents correspond to reference segments.
}

number of segments. ${ }^{7}$ We designed two test corpora, each of 500 documents, using concatenated texts extracted from the TDT and WSJ corpora, ranging from 4 to 22 in number of segments.

LCseg depends on several parameters. Parameter tuning was performed on three tuning corpora of one thousand texts each. ${ }^{8}$ We performed searches for the optimal settings of the four tunable parameters introduced above; the best performance was achieved with $h=11$ (hiatus length for dividing a chain into parts), $k=2$ (analysis window size), $p_{\text {limit }}=0.1$ and $\alpha=\frac{1}{2}$ (thresholding limits for the hypothesized boundaries).

As shown in Table 1, our algorithm is significantly better than (Choi, 2000) (labeled C99) on all three test corpora, according to a one-sided ttest of the null hypothesis of equal mean at the 0.01 level. It is not clear whether our algorithm is better than (Utiyama and Isahara, 2001) (U00). When the number of segments is provided to the algorithms, our algorithm is significantly better than Utiyama's on WSJ, better on Brown (but not significant), and significantly worse on TDT. When the number of boundaries is unknown, our algorithm is insignificantly worse on Brown, but significantly better on WSJ and TDT - the two corpora designed to have a varying number of segments per document. In the case of the Meeting corpus, none of the algorithms are significantly different than the others, due to the

\footnotetext{
${ }^{7}$ All texts in Choi's test corpus have exactly 10 segments. We are concerned that the adjustments of any algorithm parameters might overfit this predefined number of segments.

${ }^{8}$ These texts are different from the ones used for evaluation.
} 


\begin{tabular}{|c|c|c|c|c|}
\hline \multicolumn{5}{|c|}{ Brown corpus } \\
\hline & \multicolumn{2}{|c|}{ known } & unknown \\
\hline & $P_{k}$ & $W D$ & $P_{k}$ & $W D$ \\
\hline C99 & $11.19 \%$ & $13.86 \%$ & $12.07 \%$ & $14.57 \%$ \\
U00 & $8.77 \%$ & $9.44 \%$ & $9.76 \%$ & $\mathbf{1 0 . 3 2 \%}$ \\
LCseg & $8.69 \%$ & $9.42 \%$ & $10.49 \%$ & $11.37 \%$ \\
p-val. & 0.42 & 0.48 & 0.027 & 0.0037 \\
\hline \multicolumn{5}{|c|}{ TDT corpus } \\
\hline C99 & $9.37 \%$ & $11.91 \%$ & $10.18 \%$ & $12.72 \%$ \\
U00 & $\mathbf{4 . 7 0 \%}$ & 6.29\% & $8.70 \%$ & $11.12 \%$ \\
LCseg & $6.15 \%$ & $8.41 \%$ & $\mathbf{6 . 9 5 \%}$ & $\mathbf{9 . 0 9 \%}$ \\
p-val. & $1.1 \mathrm{e}-05$ & $2.8 \mathrm{e}-07$ & $4.5 \mathrm{e}-05$ & $2.8 \mathrm{e}-05$ \\
\hline \multicolumn{5}{|c|}{ WSJ corpus } \\
\hline C99 & $19.61 \%$ & $26.42 \%$ & $22.32 \%$ & $29.81 \%$ \\
U00 & $15.18 \%$ & $21.54 \%$ & $17.71 \%$ & $24.06 \%$ \\
LCseg & $\mathbf{1 2 . 2 1 \%}$ & 18.25\% & $\mathbf{1 5 . 3 1 \%}$ & $\mathbf{2 2 . 1 4 \%}$ \\
p-val. & $1.4 \mathrm{e}-08$ & $1.7 \mathrm{e}-08$ & $2.6 \mathrm{e}-04$ & 0.0063 \\
\hline \multicolumn{5}{|c|}{ Meeting corpus } \\
\hline C99 & $33.79 \%$ & $37.25 \%$ & $47.42 \%$ & $58.08 \%$ \\
U00 & $31.99 \%$ & $34.49 \%$ & $37.39 \%$ & $40.43 \%$ \\
LCseg & $26.37 \%$ & $29.40 \%$ & $31.91 \%$ & $35.88 \%$ \\
p-val. & 0.026 & 0.14 & 0.14 & 0.23 \\
\hline
\end{tabular}

Table 1: Comparison C99 and U00. The p-values in the table are the results of significance tests between U00 and LCseg. Bold-faced values are scores that are statistically significant.

small test set size.

In conclusion, LCseg has a performance comparable to state-of-the-art text segmentation algorithms, with the added advantage of computing a segmentation probability at each potential boundary. This information can be effectively used in the featurebased segmenter to account for lexical cohesion, as described in the next section.

\section{Feature-based Segmentation}

In the previous section, we have concentrated exclusively on the consideration of content (through lexical cohesion) to determine the structure of texts, neglecting any influence of form. In this section, we explore formal devices that are indicative of topic shifts, and explain how we use these cues to build a segmenter targeting conversational speech.

\subsection{Probabilistic Classifiers}

Topic segmentation is reduced here to a classification problem, where each utterance break $B_{i}$ is either considered a topic boundary or not. We use statistical modeling techniques to build a classifier that uses local features (e.g. cue phrases, pauses) to determine if an utterance break corresponds to a topic boundary. We chose $\mathrm{C} 4.5$ and $\mathrm{C} 4.5$ rules (Quinlan, 1993), two programs to induce classification rules in the form of decision trees and production rules (respectively). C4.5 generates an unpruned decision tree, which is then analyzed by C4.5rules to generate a set of pruned production rules (it tries to find the most useful subset of them). The advantage of pruned rules over decision trees is that they are easier to analyze, and allow combination of features in the same rule (feature interactions are explicit).

The greedy nature of decision rule learning algorithms implies that a large set of features can lead to bad performance and generalization capability. It is desirable to remove redundant and irrelevant features, especially in our case since we have little data labeled with topic shifts; with a large set of features, we would risk overfitting the data. We tried to restrict ourselves to features whose inclusion is motivated by previous work (pauses, speech rate) and added features that are specific to multi-speaker speech (overlap, changes in speaker activity).

\subsection{Features}

Cue phrases: previous work on segmentation has found that discourse particles like now, well provide valuable information about the structure of texts (Grosz and Sidner, 1986; Hirschberg and Litman, 1994; Passonneau and Litman, 1997). We analyzed the correlation between words in the meeting corpus and labeled topic boundaries, and automatically extracted utterance-initial cue phrases ${ }^{9}$ that are statistically correlated with boundaries. For every word in the meeting corpus, we counted the number of its occurrences near any topic boundary, and its number of appearances overall. Then, we performed $\chi^{2}$ significance tests (e.g. figure 2 for okay) under the null hypothesis that no correlation exists. We selected terms whose $\chi^{2}$ value rejected the hypothesis under a 0.01-level confidence (the rejection criterion is $\chi^{2} \geq 6.635$ ). Finally, induced cue phrases whose usage has never been described in other work were removed (marked with $*$ in Table 3 ). Indeed, there is a risk that the automatically derived list of cue phrases could be too specific to the word usage in

\footnotetext{
${ }^{9}$ As in (Litman and Passonneau, 1995), we restrict ourselves to the first lexical item of any utterance, plus the second one if the first item is also a cue word.
} 


\begin{tabular}{|c|c|c|}
\hline & Near boundary & Distant \\
\hline okay & 64 & 740 \\
Other & 657 & 25896 \\
\hline
\end{tabular}

Table 2: okay $\left(\chi^{2}=89.11, d f=1, p<0.01\right)$.

\begin{tabular}{|c|c||c|c|}
\hline okay & 93.05 & but & 13.57 \\
shall $*$ & 27.34 & so & 11.65 \\
anyway & 23.95 & and & 10.99 \\
we're $*$ & 17.67 & should $*$ & 10.21 \\
alright & 16.09 & good $*$ & 7.70 \\
let's $*$ & 14.54 & & \\
\hline
\end{tabular}

Table 3: Automatically selected cue phrases.

these meetings.

Silences: previous work has found that major shifts in topic typically show longer silences (Passonneau and Litman, 1993; Hirschberg and Nakatani, 1996). We investigated the presence of silences in meetings and their correlation with topic boundaries, and found it necessary to make a distinction between pauses and gaps (Levinson, 1983). A pause is a silence that is attributable to a given party, for example in the middle of an adjacency pair, or when a speaker pauses in the middle of her speech. Gaps are silences not attributable to any party, and last until a speaker takes the initiative of continuing the discussion. As an approximation of this distinction, we classified a silence that follows a question or in the middle of somebody's speech as a pause, and any other silences as a gap. While the correlation between long silences and discourse boundaries seem to be less pervasive in meetings than in other speech corpora, we have noticed that some topic boundaries are preceded (within some window) by numerous gaps. However, we found little correlation between pauses and topic boundaries.

Overlaps: we also analyzed the distribution of overlapping speech by counting the average overlap rate within some window. We noticed that, many times, the beginning of segments are characterized by having little overlapping speech.

Speaker change: we sometimes noticed a correlation between topic boundaries and sudden changes in speaker activity. For example, in Figure 2, it is clear that the contribution of individual speakers to the discussion can greatly change from one discourse unit to the next. We try to capture significant changes in speakership by measuring the dissimilarity between two analysis windows. For each potential boundary, we count for each speaker $i$ the number of words that are uttered before $\left(L_{i}\right)$ and after $\left(R_{i}\right)$ the potential boundary (we limit our analysis to a window of fixed size). The two distributions are normalized to form two probability distributions $l$ and $r$, and significant changes of speakership are detected by computing their Jensen-Shannon divergence:

$$
J S(l, r)=\frac{1}{2}\left[D\left(l \| a v g_{l, r}\right)+D\left(r \| a v g_{l, r}\right)\right]
$$

where $D(l \| r)$ is the KL-divergence between the two distributions.

Lexical cohesion: we also incorporated the lexical cohesion function computed by LCseg as a feature of the multi-source segmenter in a manner similar to the knowledge source combination performed by (Beeferman et al., 1999) and (Tür et al., 2001). Note that we use both the posterior estimate computed by LCseg and the raw lexical cohesion function as features of the system.

\subsection{Features: Selection and Combination}

For every potential boundary $B_{i}$, the classifier analyzes features in a window surrounding $B_{i}$ to decide whether it is a topic boundary or not. It is generally unclear what is the optimal window size and how features should be analyzed. Windows of various sizes can lead to different levels of prediction, and in some cases, it might be more appropriate to only extract features preceding or following $B_{i}$.

We avoided making arbitrary choices of parameters; instead, for any feature $F$ and a set $F_{1}, \ldots, F_{n}$ of possible ways to measure the feature (different window sizes, different directions), we picked the $F_{i}$ that is in isolation the best predictor of topic boundaries (among $F_{1}, \ldots, F_{n}$ ). Table 4 presents for each feature the analysis mode that is the most useful on the training data.

\subsection{Evaluation}

We performed 25 -fold cross-validation for evaluating the induced probabilistic classifier, computing the average of $P_{k}$ and $W D$ on the held-out meetings. Feature selection and decision rule learning 


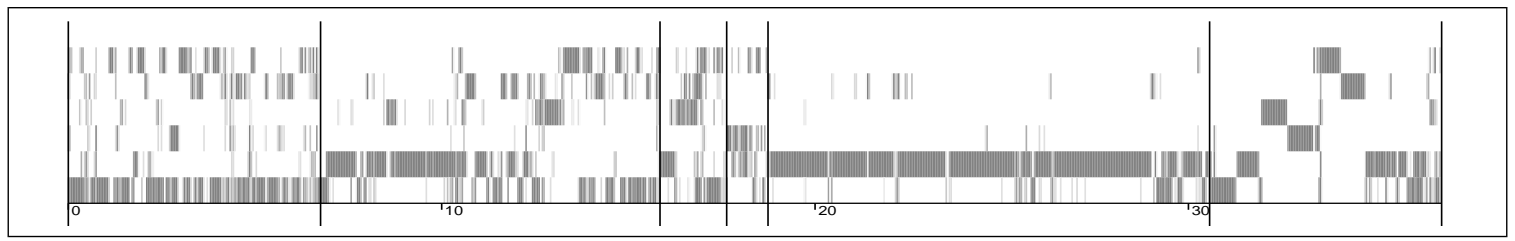

Figure 2: speaker activity in a meeting. Each row represent the speech activity of one speaker, utterance of words being represented as black. Vertical lines represent topic shifts. The $\mathrm{x}$-axis represents time.

\begin{tabular}{|c|c|c|c|}
\hline Feature & Tag & Size (sec.) & Side \\
\hline Cue phrases & CUE & 5 & both \\
Silence (gaps) & SIL & 30 & left \\
Overlap $\dagger$ & OVR & 30 & right \\
Speaker activity & ACT & 5 & both \\
Lexical cohesion & LC & 30 & both \\
\hline \\
†: the size of the window that was used to compute the \\
JS-divergence was also determined automatically.
\end{tabular}

Table 4: Parameters for feature analysis.

is always performed on sets of 24 meetings, while the held-out data is used for testing. Table 5 gives some examples of the type of rules that are learned. The first rule states that if the value for the lexical cohesion (LC) function is low at the current sentence break, there is at least one CUE phrase, there is less than three seconds of silence to the left of the break, ${ }^{10}$ and a single speaker holds the floor for a longer period of time than usual to the right of the break, then we have a topic break. In general, we found that the derived rules show that lexical cohesion plays a stronger role than most other features in determining topic breaks. Nonetheless, the quantitative results summarized in table 6 , which correspond to the average performance on the held-out sets, show that the integration of conversational features with the text-based segmenter outperforms either alone.

\section{Conclusions}

We presented a domain-independent segmentation algorithm for multi-party conversation that integrates features based on content with features based on form. The learned combination of features results in a significant increase in accuracy over previous

\footnotetext{
${ }^{10}$ Note that rules are not always meaningful in isolation and it is likely that a subordinate rule in the tree to this one would do further tests on silence to determine if a topic boundary exists.
}

\begin{tabular}{|c|c|c|}
\hline Condition & Decision & Conf. \\
\hline LC $\leq 0.67$, CUE $\geq 1$, & & \\
OVR $\leq 1.20$, SIL $\leq 3.42$ & yes & 94.1 \\
\hline $\begin{array}{c}\text { LC } \leq 0.35, \text { SIL }>3.42, \\
\text { OVR } \leq 4.55\end{array}$ & yes & 92.2 \\
\hline $\begin{array}{c}\text { CUE } \geq 1, \text { ACT }>0.1768, \\
\text { OVR } \leq 1.20, \text { LC } \leq 0.67\end{array}$ & yes & 91.6 \\
\hline \multicolumn{2}{|c|}{$\cdots$} \\
\hline default & no & \\
\hline
\end{tabular}

Table 5: A selection of the most useful rules learned by $\mathrm{C} 4.5$ rules along with their confidence levels. Times for OVR and SIL are expressed in seconds.

\begin{tabular}{|c|c|c|}
\hline & $P_{k}$ & $W D$ \\
\hline feature-based & $\mathbf{2 3 . 0 0 \%}$ & $\mathbf{2 5 . 4 7 \%}$ \\
\hline LCseg & $31.91 \%$ & $35.88 \%$ \\
U00 & $37.39 \%$ & $40.43 \%$ \\
\hline p-value & $2.14 \mathrm{e}-04$ & $3.30 \mathrm{e}-04$ \\
\hline
\end{tabular}

Table 6: Performance of the feature-based segmenter on the test data.

approaches to segmentation when applied to meetings. Features based on form that are likely to indicate topic shifts are automatically extracted from speech. Content based features are computed by a segmentation algorithm that utilizes a metric of lexical cohesion and that performs as well as state-ofthe-art text-based segmentation techniques. It works both with written and spoken texts. The text-based segmentation approach alone, when applied to meetings, outperforms all other segmenters, although the difference is not statistically significant.

In future work, we would like to investigate the effects of adding prosodic features, such as pitch ranges, to our segmenter, as well as the effect of using errorful speech recognition transcripts as op- 
posed to manually transcribed utterances.

An implementation of our lexical cohesion segmenter is freely available for educational or research purposes. ${ }^{11}$

\section{Acknowledgments}

We are grateful to Julia Hirschberg, Dan Ellis, Elizabeth Shriberg, and Mari Ostendorf for their helpful advice. We thank our ICSI project partners for granting us access to the meeting corpus and for useful discussions. This work was funded under the NSF project Mapping Meetings (IIS-012196).

\section{References}

D. Beeferman, A. Berger, and J. Lafferty. 1999. Statistical models for text segmentation. Machine Learning, 34(1-3):177-210.

F. Choi. 2000. Advances in domain independent linear text segmentation. In Proc. of NAACL'OO.

W. Cochran. 1950. The comparison of percentages in matched samples. Biometrika, 37:256-266.

B. Grosz and J. Hirschberg. 1992. Some intonational characteristics of discourse structure. In Proc. of ICSLP-92, pages 429-432.

B. Grosz and C. Sidner. 1986. Attention, intentions and the structure of discourse. Computational Linguistics, 12(3).

M. Hajime, H. Takeo, and O. Manabu. 1998. Text segmentation with multiple surface linguistic cues. In COLING-ACL, pages 881-885.

M. Hearst. 1994. Multi-paragraph segmentation of expository text. In Proc. of the ACL.

J. Hirschberg and D. Litman. 1994. Empirical studies on the disambiguation of cue phrases. Computational Linguistics, 19(3):501-530.

J. Hirschberg and C. Nakatani. 1996. A prosodic analysis of discourse segments in direction-giving monologues. In Proc. of the ACL.

J. Hirschberg and C. Nakatani. 1998. Acoustic indicators of topic segmentation. In Proc. of ICSLP.

A. Janin, D. Baron, J. Edwards, D. Ellis, D. Gelbart, N. Morgan, B. Peskin, T. Pfau, E. Shriberg, A. Stolcke, and C. Wooters. 2003. The ICSI meeting corpus. In Proc. of ICASSP-03, Hong Kong (to appear).

\footnotetext{
${ }^{11} \mathrm{http}: / / \mathrm{www} . c s . c o l u m b i a . e d u /$ galley/research.html
}

M.-Y. Kan, J. Klavans, and K. McKeown. 1998. Linear segmentation and segment significance. In Proc. 6th Workshop on Very Large Corpora (WVLC-98).

H. Kozima. 1993. Text segmentation based on similarity between words. In Proc. of the ACL.

S. Levinson. 1983. Pragmatics. Cambridge University Press.

D. Litman and R. Passonneau. 1995. Combining multiple knowledge sources for discourse segmentation. In Proc. of the ACL.

J. Morris and G. Hirst. 1991. Lexcial cohesion computed by thesaural relations as an indicator of the structure of text. Computational Linguistics, 17:21-48.

C. Nakatani, J. Hirschberg, and B. Grosz. 1995. Discourse structure in spoken language: Studies on speech corpora. In AAAI-95 Symposium on Empirical Methods in Discourse Interpretation.

R. Passonneau and D. Litman. 1993. Intention-based segmentation: Human reliability and correlation with linguistic cues. In Proc. of the ACL.

R. Passonneau and D. Litman. 1997. Discourse segmentation by human and automated means. Computational Linguistics, 23(1):103-139.

L. Pevzner and M. Hearst. 2002. A critique and improvement of an evaluation metric for text segmentation. Computational Linguistics, 28 (1):19-36.

R. Quinlan. 1993. C4.5: Programs for Machine Learning. Machine Learning. Morgan Kaufmann.

J. Reynar. 1994. An automatic method of finding topic boundaries. In Proc. of the ACL.

J. Reynar. 1999. Statistical models for topic segmentation. In Proc. of the ACL.

G. Salton and C. Buckley. 1988. Term weighting approaches in automatic text retrieval. Information Processing and Management, 24(5):513-523.

G. Tür, D. Hakkani-Tür, A. Stolcke, and E. Shriberg. 2001. Integrating prosodic and lexical cues for automatic topic segmentation. Computational Linguistics, 27(1):31-57.

M. Utiyama and H. Isahara. 2001. A statistical model for domain-independent text segmentation. In Proc. of the ACL.

J. Xu and B. Croft. 1998. Corpus-based stemming using cooccurrence of word variants. ACM Transactions on Information Systems, 16(1):61-81. 